\title{
AVALIAÇÃO DO RISCO NA CADEIA DE SUPRIMENTO: UM ESTUDO EXPLORATÓRIO NO SETOR ALIMENTÍCIO
}

\section{RISK ASSESSMENT IN THE SUPPLY CHAIN: A STUDY IN THE FOOD SECTOR EXPLORATION}

\author{
Liane Márcia Freitas Silva ${ }^{* * *}$ E-mail: lianemarcia@hotmail.com \\ Ana Camila Rodrigues de Oliveira* E-mail: camila lynn@hotmail.com \\ Maria Silene Alexandre Leite* E-mail: mariasileneleite@hotmail.com \\ Fernando Augusto Silva Marins ${ }^{* *}$ E-mail: fmarins@feg.unesp.br \\ * Universidade Federal da Paraíba (UFPB), João Pessoa, PB \\ ** Universidade Estadual Paulista "Júlio de Mesquita Filho" (UNESP), São Paulo, SP
}

\begin{abstract}
Resumo: Este artigo apresenta a avaliação dos riscos em duas grandes empresas participantes de duas cadeias de suprimentos do setor alimentício. A partir do levantamento teórico, utilizou-se um questionário baseado em 24 classificações de riscos para mapear os riscos inerentes às empresas e suas repercursões nas cadeias de suprimentos ás quais pertencem. Os questionários foram respondidos por individuos do nível gerencial devido ao amplo conhecimento que possuem. Posteriormente, realizou-se uma análise dos riscos por meio de matrizes que utilizam as dimensões de frequência de ocorrência e impacto do risco. Os principais resultados apontaram que as empresas pesquisadas apresentam riscos semelhantes no que se refere aos riscos internos, como o risco de demanda, de capacidade, de informação e o risco operacional. Isso mostra que as empresas investigadas possuem ainda dificuldades na gestão que podem afetar o seu negócio. Observou-se também que a quantidade de riscos externos à empresa foram em menor número e os riscos externos à cadeia inexistentes. Por fim, os riscos mais críticos para as duas empresas foram levantados, sendo que para a empresa A estes foram: o risco operacional, de estoque e de preço, enquanto que para a empresa $\mathrm{B}$ foram: o risco de preço, logístico, cliente e operacional. Dessa forma, pode-se concluir que a avaliação dos riscos, apesar de ser de natureza qualitativa, é fundamental para estabelecer as estratégias de mitigação apropriadas.
\end{abstract}

Palavras-chave: Riscos. Avaliação de Riscos. Gestão de Riscos na Cadeia de Suprimentos. Setor Alimentício.

Abstract: This article presents the risk assessment in two major companies participating in two food supply chain. From the theoretical survey, a questionnaire was used based on 24 ratings risks to map the risks to companies and their repercussions in the supply chains to which they belong. The questionnaires were answered by the management level individuals due to the extensive knowledge they possess. Later, there was a risk analysis through arrays using the frequency of occurrence of dimensions and impact of the risk. The main results showed that the surveyed companies have similar risks in relation to internal risks such as the risk of demand, capacity, information and operational risk. This shows that the investigated companies still have difficulties in managing that can affect your business. It was also observed that the amount of external risks to the company was fewer and external risks to non-existent chain. Finally, the most critical risks for both companies were raised, and for the company to these were: operational risk, inventory and price, while for Company B were: price risk, logistics, customer and operating. Thus, it can be concluded that the risk assessment, although qualitative, it is essential to establish the appropriate mitigation strategies.

Keywords: Risks. Risk Assessment. Risk Management In The Supply Chain. Food Industry. 


\section{INTRODUÇÃO}

As cadeias de suprimento tornaram-se importantes elementos da economia global (WU; OLSON, 2008). Pela gama de fluxos e informações a serem gerenciadas, Tang (2006) aponta que a gestão de uma cadeia de suprimento é uma atividade de alta complexidade. A complexidade das cadeias de suprimentos tem uma influência direta sobre os riscos (PUNNIYAMOORTHY; THAMARAISELVAN; MANIKANDAN, 2013).

Além da complexidade inerente às cadeias de suprimentos, a vulnerabilidade é aumentada por eventos ambientais, sociopolíticos e econômicos. Sodhi, Son e Tang (2012) alertam que eventos como o atentado de 11 de Setembro, o furacão Katrina de 2005 e o tsunami no Japão em 2011 mostraram que as cadeias de suprimentos globais estão expostas a eventos inesperados, e por isso, devem também planejar como atuar diante de tais acontecimentos.

Tang (2006) ainda relata que as interrupções em cadeias de suprimentos podem ter impacto negativo direto no desempenho financeiro das organizações. $O$ autor cita o caso da Ericsson, que perdeu 400 milhões de euros após um incêndio na fábrica do seu fornecedor de semicondutores no ano 2000.

Norrman e Jansson (2004) e Juttner (2005) alertam que eventos como incêndio, protestos, greves, doenças, crises econômicas e furacões podem paralisar a produção de empresas, ocasionando descontinuidade de produção.

Neste contexto, diversos modelos de gestão de riscos para as cadeias de suprimentos tem sido elaborados, construindo uma teoria com o objetivo de mitigar os riscos, seja pela eliminação dos riscos ou pelo aumento da resiliência da cadeia. Esses modelos compõem um campo de gestão denominado de Supply Chain Risk Management (SCRM) que preocupa-se em gerir os riscos a que estão expostas as cadeias de suprimentos e garantir a rentabilidade e a continuidade dos processos logísticos que ocorrem nas cadeias de suprimentos (TANG, 2006).

De uma maneira geral, o risco é compreendido como uma relação entre severidade e frequência de ocorrência (NORRMAN; JANSSON, 2004; RITCHIE; BRINDLEY, 2007; WANG; YANG, 2007; TRKMAN; MCCORMACK, 2009; LOCKAMY; MCCORMACK, 2010; TANG; MUSA, 2011; TUMMALA; SCHOENHERR, 2011; WIELAND, 2013; KUMAR; HIMES; KRITZER, 2014). 
No sentido de avaliar os riscos na cadeia de suprimentos foi feita uma investigação em duas empresas do setor de alimentos, no qual uma delas possui parte do fornecimento verticalizado, enquanto a outra possui uma cadeia de suprimentos totalmente desverticalizada a montante e a jusante. A partir de entrevistas diretas com os especialistas que fazem parte do nível gerencial das empresas foram mapeados os riscos e as variáveis que permitiram avaliar a criticidade dos riscos. Na sequência, foi realizada a avaliação dos riscos identificados de modo a direcionar as empresas para as medidas de mitigação que poderiam ser adotadas para tratar os riscos prioritários.

Este artigo está organizado com a seguinte estrutura: Seção 2- é feita uma explanação sobre a gestão de riscos na cadeia de suprimentos; Seção 3- o método de pesquisa é descrito; Seção 4- os resultados da pesquisa empírica são apresentados e discutidos, e Seção 5 - as considerações principais sobre os resultados são pontuadas.

\section{GESTÃO DE RISCOS NA CADEIA DE SUPRIMENTOS}

Bode e Wagner (2015) afirmam que as cadeias de suprimentos tornaram-se mais complexas nas últimas décadas, no entanto, esta não é uma característica desejável, devendo haver um esforço por parte da cadeia para gerenciar este ambiente de alta vulnerabilidade. Christopher e Lee (2004) concordam afirmando que é crescente a exposição das cadeias de suprimentos aos riscos, e por isso, enxergam a necessidade de novas respostas para gerenciar estas estruturas.

A fim de minimizar os efeitos dos riscos ou mesmo eliminar a ocorrência dos riscos Fazli, Mavi e Vosooghidizaji (2015) enfatizam a necessidade de adotar a gestão de riscos na cadeia de suprimentos, pois esta desempenha um papel importante para diminuir os desvios inesperados, aumentando as chances de consistência em relação às metas financeiras e de produção.

Nesse sentido, para manter a lucratividade, as cadeias de suprimentos devem ser capazes de responder rapidamente a eventos de riscos internos e externos e manter seus negócios eficientes e dinâmicos (AQLAN; LAM, 2015).

Hauser (2003) e Manuj e Mentzer (2008) indicam que elaborar plano de gestão de risco para as cadeias de suprimentos pode se traduzir em um melhor desempenho financeiro e competitivo. Diante de tais percepções, as organizações 
devem adotar estratégias eficazes de gestão dos riscos, de modo a reduzir a probabilidade de ocorrência destes, bem como o impacto caso estes ocorram.

Khan e Burnes (2007) indicam que a gestão de riscos na cadeia de suprimentos pode ser compreendida como modelo de gestão metódico que deve abordar todos os riscos que cercam as atividades da organização no passado, no presente e em particular, do futuro. Deve-se assim, tentar evitar tais eventos e desenvolver estratégias para gerenciar o impacto relacionado aos riscos.

Autry e Bobbitt (2008) afirmam que a gestão de riscos na cadeia de supirmentos inclui os processos utilizados para reduzir a probabilidade de ocorrência do risco e/ou o impacto que estes eventos podem causar de maneira a prejudicar toda a cadeia. Segundo Lockamy e McCormack (2010), a SCRM é um processo formal que visa desenvolver abordagens para identificação, avaliação, análise e tratamento de áreas de vulnerabilidade e risco em cadeias de suprimentos. De forma análoga, Sun, Matsui e Yin (2012) indicam que os objetivos do SCRM são controlar, monitorar e avaliar os riscos da cadeia de suprimentos, de modo a assegurar a continuidade e maximizar a rentabilidade das empresas.

Mais recentemente, Wiengarten et al (2015) e Aqlan e Lam (2015) concluem que SCRM é um processo integrado e sistemático para identificar, avaliar e mitigar as incertezas e os riscos na cadeia de suprimentos, monitorando as possíveis interrupções na cadeia de suprimento, a fim de reduzir o impacto negativo dessas interrupções nas operações da cadeia.

De maneira geral, os modelos de gestão de riscos na cadeia de suprimentos, de acordo com Cohen e Kunreuther (2007), Giannakis e Louis (2011) e Tummala e Schoenherr (2011) são formatadas por quatro etapas de gestão: 1- identificação dos riscos; 2- avaliação dos riscos; 3- proposição de estratégias para aceitar, transferir, reduzir ou eliminar os riscos; e por fim, 4- monitoramento de seus impactos. Segundo esses autores, ao cumprir estas etapas, devem ser apontadas estratégias para mitigação dos riscos, que podem incluir estratégias para reduzir/eliminar o impacto dos riscos ou a probabilidade de ocorrência.

O modelo pioneiro de Harland, Brenchley e Walker (2003) é bastante sintético quando comparado aos modelos de gestão de riscos na cadeia de suprimentos mais recentes. Para estes autores, a gestão de riscos é composta pelas fases de 
identificação e avaliação dos riscos, seguida pela proposição de estratégias para combater estes riscos.

Outro modelo pioneiro foi o proposto por Hallikas et al (2004). Na visão de tais autores, um modelo de gestão de riscos na cadeia de suprimentos deve ser executado em quatro grandes etapas: 1- identificação dos riscos; 2- avaliação dos riscos; 3- proposição de estratégias para aceitar, transferir, reduzir ou eliminar os riscos; e por fim, 4- monitoramento de seus impactos.

De maneira geral, sobre os modelos de gestão de riscos em cadeia de suprimentos é possível identificar que o modelo de gestão para cadeia de suprimentos proposto por Hallikas et al (2004) serviu de base para outros modelos mais recentes, como os de Cohen e Kunreuther (2007), Giannakis e Louis (2011), Olson e Wu (2011), Blome e Schoenherr (2011) e Tummala e Schoenherr (2011).

Rao e Goldsby (2009) apontam o trabalho de Jüttner, Peck e Christopher (2003) como referência de modelo de SCRM, identificando este como um dos trabalhos pioneiros na classificação dos riscos. Na proposta de modelo de gestão desenvolvida por Jüttner, Peck e Christopher (2003) foi sugerido um modelo composto por quatro fases: 1-identificação das fontes de risco para a cadeia de suprimentos; 2- avaliação dos riscos; 3- proposiçao de estratégias, e 4-mitigação dos riscos para a cadeia.

O modelo de Gaonkar e Viswanadham (2004) contempla as fases de 1identificação das fontes de riscos; 2- identificação dos fatores de riscos; 3- avaliação dos riscos, e por fim, 4- proposição de estratégias de tratamento aos riscos. Ritchie e Brindley (2007b) e Matook, Lasch e Tamaschke (2009) propõem cinco fases: 1identificação dos riscos, 2- definição dos indicadores de desempenho para tomada de decisão em relação ao risco, 3- desenvolvimento de ações em resposta aos riscos, 4- medição dos resultados, e por fim, 5- mensuração dos ganhos.

Trkman e McCormack (2009) propuseram alguns passos para a gestão dos riscos: 1- identificação de riscos, 2- avaliação dos riscos e 3- gerenciamento e monitoramento de risco. Blackhurst, Scheibe e Johnson (2008) desenvolveram um modelo bastante prático que foi aplicado em uma empresa do setor automobilístico, cujo objetivo principal era monitorar os riscos da cadeia de suprimento relativos aos fornecedores. Este modelo contou com as fases de identificação dos riscos e avaliação dos riscos. 
Por fim, acredita-se ser importante ressaltar o modelo de gestão proposto pela ISO 31000:2009, que reconhece que um modelo de gestão de riscos deve ser um processo global que se inicia pela 1-identificação de riscos, 2- análise de riscos, 3- avaliação de riscos, e por fim, 4- monitoramento dos riscos.

Descrevendo as fases para aplicação de um modelo de gestão de riscos na cadeia de suprimentos tem-se que a identificação dos riscos na cadeia de suprimentos é o primeiro passo para o processo de gestão de riscos (PFOHL; GALLUS; THOMAS, 2011; HO et al, 2015).

Os modelos, em sua maioria, começam com a fase de identificação dos riscos, para que se conheça o que se deve gerenciar seguindo para a avaliação do risco, momento onde a criticidade do risco é medida, oportunidade onde os riscos mais críticos são hierarquizados.

O modelo de gestão da ISO 31000:2009 explica que a análise de riscos objetiva compreender a natureza do risco e determinar o nível de risco, ou seja, estimar a ocorrência do risco bem como a magnitude de seus impactos. Em contrapatida, a avaliação do risco compreende o processo de comparar os resultados da análise de riscos em relação aos critérios de risco a fim de determinar se o risco ou sua magnitude é aceitável, inaceitável ou tolerável.

De forma resumida Purdy (2010) aponta que a avaliação de risco objetiva realizar uma compreensão de cada risco, suas consequências e da probabilidade dessas consequências, enquanto a avaliação de riscos envolve a tomada de uma decisão sobre o nível de risco e a prioridade segundo critérios estabelecidos pela organização. Na avaliação de riscos, as empresas devem se concentrar na adição de estratégias para mitigação dos riscos (HALLIKAS et al, 2004).

Hallikas et al (2004) esclarecem que a avaliação dos riscos pode ser feita por meio de escalas, onde a probabilidade e o impacto do risco são avaliados conforme disposto nas Tabelas 1 e 2 , respectivamente.

Com as informações obtidas sobre a probabilidade e o impacto do risco, é possível construir um diagrama de risco (BLACKHURST; SCHEIBE; JOHNSON, 2008; HALLIKAS et al, 2004), conforme ilustrado na Figura 1. 
Tabela 1- Escala para avaliação da probabilidade do risco

\begin{tabular}{l|l|l}
\hline Ranking & Estimação & Descrição \\
\hline 1 & Muito improvável & Evento muito raro \\
\hline 3 & Improvável & Existe evidência indireta do evento \\
\hline 4 & Moderado & Existe evidência direta do evento \\
\hline 5 & Provável & Existe evidência direta forte do evento \\
\hline
\end{tabular}

Fonte - Hallikas et al. (2004)

Tabela 2 - Escala para avaliação do impacto do risco

\begin{tabular}{l|l|l}
\hline Ranking & Estimação & Descrição \\
\hline 1 & Nenhum impacto & Insignificante em termos de toda empresa \\
\hline 2 & Pouco impacto & Pequenas perdas \\
\hline 3 & Impacto médio & Causa dificuldades de curto prazo \\
\hline 4 & Impacto sério & Causa dificuldades de longo prazo \\
\hline 5 & Impacto catastrófico & Descontinuidade dos negócios \\
\hline
\end{tabular}

Fonte - Hallikas et al. (2004)

Figura 1 - Diagrama de risco

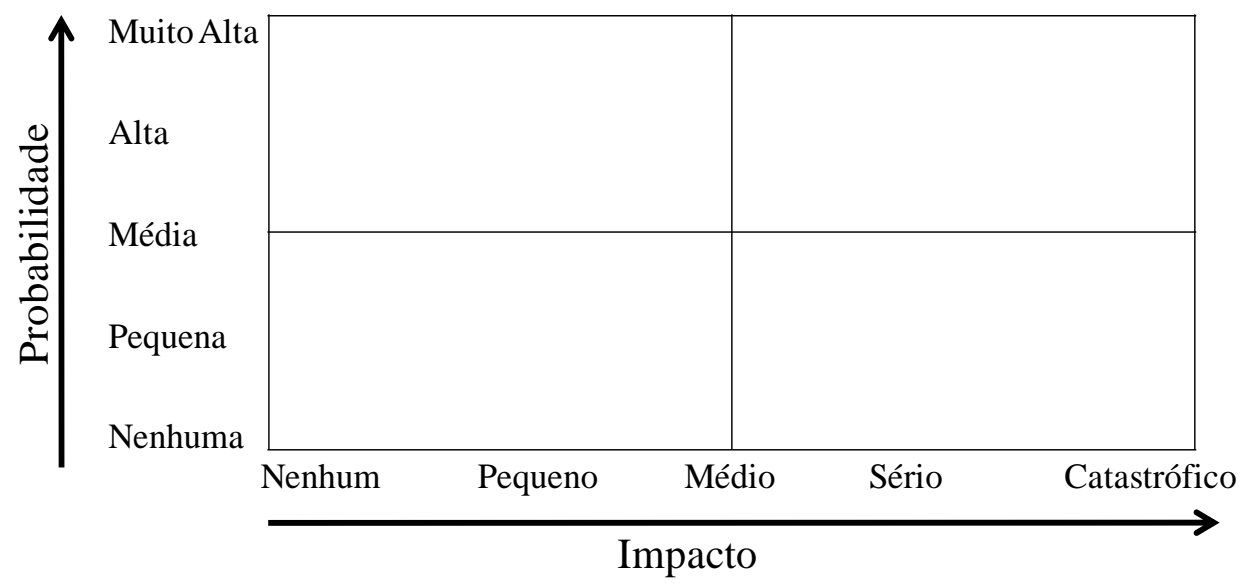

Fonte - Blackhurst, Scheibe e Johnson (2008)

O diagrama de risco permite uma visualização dos riscos, onde os que são considerados mais importantes são mais visíveis. Percebe-se assim que um risco pode possuir alta probabilidade de ocorrência e baixo impacto, no entanto, outro determinado risco pode possuir alta probabilidade de ocorrência e alto impacto e, nessa perspectiva, as organizações devem ser capazes de avaliar quais são os riscos com potencial de gerar consequências mais graves.

Sobre a avaliação dos riscos, Aqlan e Lam (2015) apontam que embora o uso de dados históricos seja recomendado para compreender a magnitude do risco,

Revista Produção Online, Florianópolis, SC, v. 17, n. 1, p. 351-375, jan./mar. 2017. 
esses dados podem ser inexistentes ou incompletos. Neses casos, quando não há dados suficientes para utilizar um método quantitativo, pode-se aplicar um método qualitativo para a avaliação dos riscos, como por exemplo os diagramas de risco.

O quadro 1 apresenta, de forma resumida, a decrição de cada etapa do processo para gestão de riscos, identificação, avaliação, mitigação e monitoramento dos riscos a partir do levantamento teórico realizado.

Quadro 1 - Etapas da gestão de riscos

\begin{tabular}{|c|c|c|}
\hline Etapa & Descrição & Autores \\
\hline $\begin{array}{l}\text { Identificação } \\
\text { do risco }\end{array}$ & $\begin{array}{l}\text { - Primeiro passo para entender as fontes de riscos (tanto } \\
\text { internas quanto externas) que as cadeias de suprimentos } \\
\text { estão submetidas; } \\
\text { - Para identificar os riscos de forma eficaz, uma } \\
\text { classificação de riscos é bastante útil, uma vez que } \\
\text { permitirá que uma determinada organização reconheça os } \\
\text { riscos a que está submetida. }\end{array}$ & $\begin{array}{l}\text { Ho et al. (2015); } \\
\text { Kern et al. (2012); } \\
\text { Adhitya, Srinivasan } \\
\text { e Karimi (2009); Shi } \\
\text { (2004) }\end{array}$ \\
\hline $\begin{array}{l}\text { Avaliação do } \\
\text { risco }\end{array}$ & $\begin{array}{l}\text { - O principal objetivo da avaliação dos riscos é auxiliar a } \\
\text { compreensão dos fatores que levam à ocorrência de um } \\
\text { risco específico, ao mesmo tempo em que fornece } \\
\text { informações sobre o impacto destes, a fim de que se possa } \\
\text { evitá-los ou reduzir o efeito de suas consequências. } \\
\text { - Etapa da gestão de riscos, onde os eventos de risco } \\
\text { precisam ser priorizados de modo que os planos de } \\
\text { mitigação de risco sejam determinados. }\end{array}$ & $\begin{array}{l}\text { Tomas e Alcantara } \\
\text { (2013); Ahmed et al. } \\
\text { (2007) }\end{array}$ \\
\hline $\begin{array}{l}\text { Mitigação do } \\
\text { risco }\end{array}$ & $\begin{array}{l}\text { - Estratégias de mitigação do risco na cadeia de } \\
\text { suprimentos são ações com a finalidade de mitigar as } \\
\text { incertezas anteriormente identificadas; } \\
\text { - Os dados coletados nas etapas anteriores do processo } \\
\text { de gestão de riscos são utilizados para estabelecer as } \\
\text { medidas adequadas. Isso inclui estratégias clássicas de } \\
\text { mitigação (antes do evento de risco) e planos de } \\
\text { contingência (após a ocorrência do evento de risco. }\end{array}$ & $\begin{array}{c}\text { Ceryno, Scavarda e } \\
\text { Klingebiel (2014); } \\
\text { Kern et al. (2012). }\end{array}$ \\
\hline $\begin{array}{l}\text { Monitoramento } \\
\text { do risco }\end{array}$ & $\begin{array}{l}\text { - O monitoramento contínuo dos riscos faz-se necessário } \\
\text { não apenas para controle do risco, mas também para a } \\
\text { análise da eficácia das estratégias adotadas, e ajuste de } \\
\text { medidas quando isso se fizer necessário; } \\
\text { - Ajuda a lançar luz sobre as áreas potenciais de melhoria } \\
\text { e reconhece a contribuição de medidas eficazes tomadas, } \\
\text { bem como as lições aprendidas a partir de incidentes } \\
\text { anteriores, }\end{array}$ & Kern et al. (2012) \\
\hline
\end{tabular}

Fonte:Elaboração própria (2016)

Neste cenário, o objetivo deste artigo é apresentar uma avaliação dos riscos em duas empresas do setor alimentício que fazem parte de duas distintas cadeias de suprimentos. A descrição do método adotado é descrito a seguir. 


\section{MÉTODO DA PESQUISA}

Inicialmente foram mapeados os riscos em duas empresas selecionadas para o estudo. Estas empresas fazem parte de duas cadeias de suprimentos distintas que fazem parte do setor alimentício. Foram levantados dados sobre a criticidade dos riscos, severidade e frequência de ocorrência dos riscos. Posteriormente, realizou-se a avaliação dos riscos identificados de modo a direcionar as empresas para a mitigação dos riscos prioritários. O fluxo metodológico seguido para a realização desta pesquisa é apresentado na Figura 2.

Figura 2 - Fluxo metodológico para realização da pesquisa

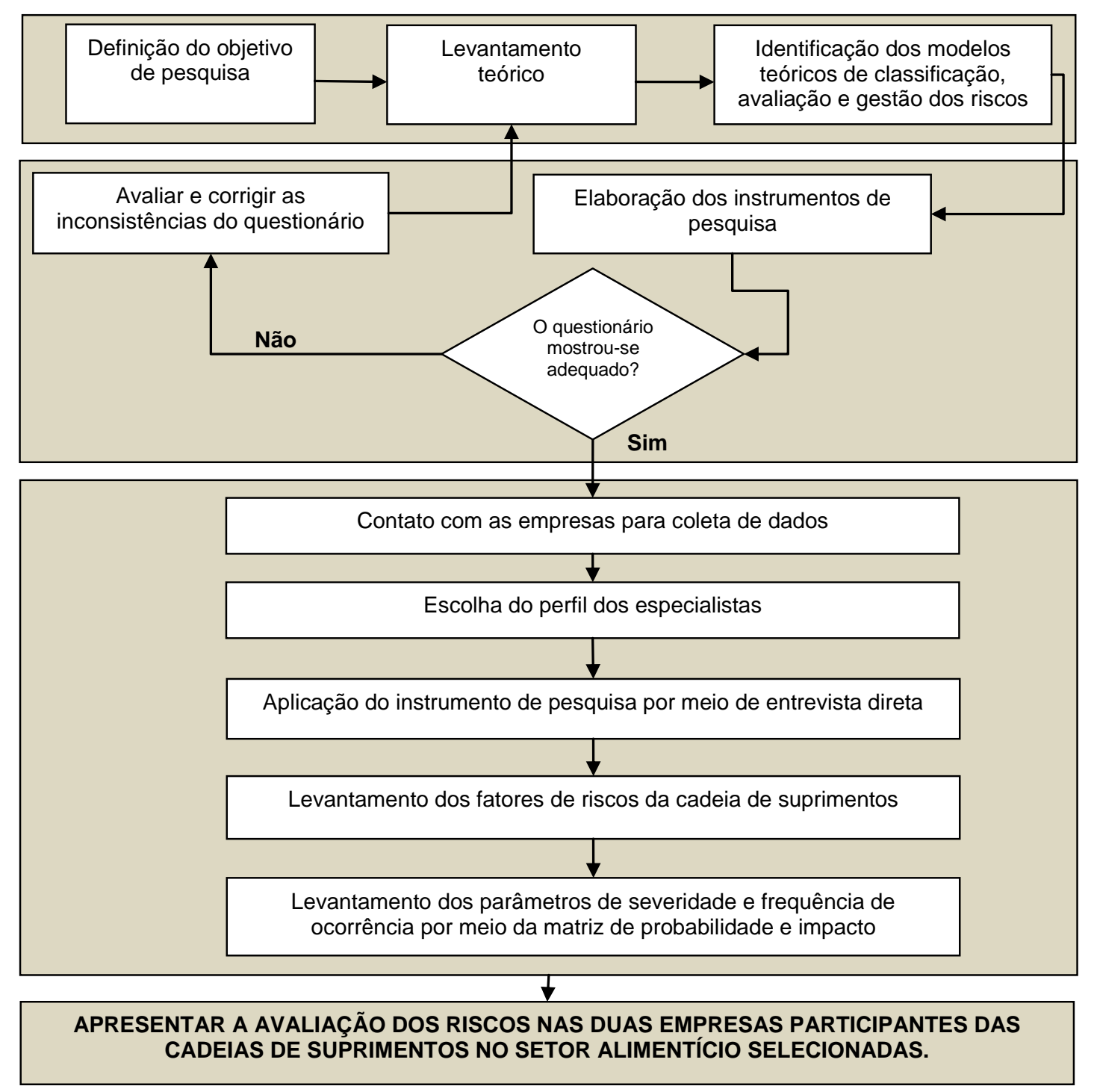

Fonte: Elaboração própria (2016) 
Assim, esta pesquisa propõe uma avaliação dos riscos em duas empresas que fazem parte de duas cadeias de suprimentos distintas do setor alimentício, sob a perspectiva da empresa focal, pois a identificação dos riscos e seus parâmetros de criticidade foram mapeados apenas na empresa focal da cadeia.

A adoção deste método limita os resultados alcançados, pois não podem ser generalizados para todo setor alimentício, mas permitem uma primeira avaliação dos riscos para estas cadeias de suprimentos e para este setor industrial, podendo ser ampliados por pesquisas futuras a fim de que os resultados possam ser mais representativos.

A avaliação dos riscos nas empresas das duas cadeias de suprimentos investigadas seguiu algumas etapas que se assemelham às fases de gestão dos modelos de gestão de riscos em cadeia de suprimentos Hallikas et al (2004), Cohen e Kunreuther (2007), Giannakis e Louis (2011), Olson e Wu (2011), Blome e Schoenherr (2011) e Tummala e Schoenherr (2011).

Foram adotadas as fases de identificação, avaliação e mitigação dos riscos. Entende-se que o delineamento do monitoramento dos riscos deve ser feito após o estabelecimento destas três primeiras etapas. Adotou-se um método que utilizou dois estudos de caso em duas empresas de um mesmo setor, que possuem características semelhantes de porte, tempo de funcionamento e cultura de gestão, mas que assumem graus de verticalização a montante distintos e compõem cadeias de suprimentos distintas.

Assim, a pesquisa inicia-se pela identificação dos riscos nas cadeias de suprimentos. Nesta etapa, os riscos são identificados por meio de um questionário construído a partir das diversas classificações de riscos existentes na literatura, conforme quadro 2.

Com o objetivo de facilitar o entendimento dos respondentes sobre o risco abordado, cada risco foi fragmentado em fatores de riscos, conforme quadro 2. Dessa forma, considerou-se que se um fator de risco ligado a uma categoria de risco for identificado, então o risco existe na empresa investigada. O preenchimento dos questionários foi efetuado pelo pesquisador, após a leitura da pergunta com suas respectivas opções de resposta. Nesse sentido, a resposta verbal dada pelo entrevistado foi preenchida pelo pesquisador. 
Salienta-se que o questionário apresentado é um recorte e, dessa forma, todos os 24 riscos investigados não estão presentes no quadro citado, que foram: risco ambiental, risco de segurança, riscos político/social, risco de propriedade intelectual, risco de preço, risco de reputação, risco comportamental, risco legal, risco estratégico, risco de mercado, risco de processo/controle, risco de demanda, risco de capacidade, risco operacional, risco de fornecimento, risco logístico, risco de estoque, risco tecnológico, risco de informação, risco de cliente, risco cultural, risco de desing, risco econômico/financeiro e risco fiscal.

O questionário utilizado nesta pesquisa é um instrumento que ainda está em fase de teste e os resultados apresentados neste estudo resumem os resultados de parte desta fase de teste. No entanto, ressalta-se que este instrumento será utilizado como questionário para identificação dos riscos em uma pesquisa de doutorado em desenvolvimento na área de gestão de riscos da cadeia de suprimentos.

Por isso, o número de respondentes e de amostra é relativamente reduzido, pois traduz esse esforço de construção e teste deste instrumento de pesquisa. Apesar dessa limitação, o instrumento de pesquisa utilizado e sua aplicação em duas empresas do setor de alimentos indicam resultados significativos quer seja pelo teste do instrumento de pesquisa, como também pela resultados práticos alcançados, pois foi possível um primeira investigação sobre os riscos que ocorrem nas duas cadeias de suprimentos e uma avaliação destes riscos. 
Quadro 2 - Recorte do questionário utilizado para identificação e avaliação dos riscos

\begin{tabular}{|c|c|c|c|c|c|c|}
\hline \multirow{2}{*}{ TIPOS DE RISCOS } & \multirow{2}{*}{ FATORES DE RISCOS } & \multicolumn{3}{|c|}{ Existe? } & \multirow{2}{*}{$\begin{array}{l}\text { Probabilidade } \\
\text { de ocorrência }\end{array}$} & \multirow{2}{*}{$\begin{array}{l}\text { Qual o impacto do } \\
\text { risco? }\end{array}$} \\
\hline & & SIM & NÁO & NÃO SEl & & \\
\hline \multirow{3}{*}{ AMBIENTAL } & $\begin{array}{l}\text { 1. Tempestades já afetaram a capacidade da empresa de atender } \\
\text { seus clientes? }\end{array}$ & & & & \multirow{3}{*}{$\begin{array}{l}\text { 1) Alta } \\
\text { 2) Média } \\
\text { 3) Baixa }\end{array}$} & \multirow{3}{*}{$\begin{array}{l}\text { 1) Severo } \\
\text { 2) Alto } \\
\text { 3) Médio } \\
\text { 4) Pequeno } \\
\text { 5) Nenhum impacto }\end{array}$} \\
\hline & $\begin{array}{l}\text { 2. Deslizamentos ou soterramentos já afetaram a capacidade da } \\
\text { empresa atender seus clientes? }\end{array}$ & & & & & \\
\hline & $\begin{array}{l}\text { 3. Incêndios já afetaram a capacidade da empresa de atender seus } \\
\text { clientes? }\end{array}$ & & & & & \\
\hline \multirow{7}{*}{ SEGURANÇA } & 1. A ocorrência de roubos já afetou negativamente a empresa? & & & & \multirow{6}{*}{$\begin{array}{l}\text { 1) Alta } \\
\text { 2) Média } \\
\text { 3) Baixa }\end{array}$} & \multirow{6}{*}{$\begin{array}{l}\text { 1) Severo } \\
\text { 2) Alto } \\
\text { 3) Médio } \\
\text { 4) Pequeno } \\
\text { 5) Nenhum impacto }\end{array}$} \\
\hline & 2. A ocorrência de vandalismo já afetou negativamente a empresa? & & & & & \\
\hline & 3. Ameaças terroristas já afetaram o funcionamento da empresa? & & & & & \\
\hline & $\begin{array}{l}\text { 4. Ocorrências de sabotagem (por concorrentes/funcionários) já } \\
\text { afetaram negativamente a empresa? }\end{array}$ & & & & & \\
\hline & $\begin{array}{l}\text { 5. A ocorrência de crimes envolvendo pessoas da empresa ou nas } \\
\text { dependências da empresa já afetou negativamente a empresa? }\end{array}$ & & & & & \\
\hline & $\begin{array}{l}\text { 6. A ocorrência de acidentes de trabalho envolvendo pessoas da } \\
\text { empresa ou nas dependências da empresa já afetou negativamente } \\
\text { a empresa? }\end{array}$ & & & & & \\
\hline & $\begin{array}{l}\text { 7. Invasão de hackers nos sistemas de informação já afetou o } \\
\text { funcionamento da empresa? }\end{array}$ & & & & \multirow{4}{*}{$\begin{array}{l}\text { 1) Alta } \\
\text { 2) Média } \\
\text { 3) Baixa }\end{array}$} & \multirow{4}{*}{$\begin{array}{l}\text { 1) Severo } \\
\text { 2) Alto } \\
\text { 3) Médio } \\
\text { 4) Pequeno } \\
\text { 5) Nenhum impacto }\end{array}$} \\
\hline \multirow{3}{*}{ SOCIAL } & $\begin{array}{l}\text { 1. Movimentos sociais como greves já afetaram o funcionamento da } \\
\text { empresa? }\end{array}$ & & & & & \\
\hline & 2. Manifestações sociais como protestos já afetaram a empresa? & & & & & \\
\hline & $\begin{array}{l}\text { 3. Movimentos sociais como ações sindicais já afetaram a } \\
\text { empresa? }\end{array}$ & & & & & \\
\hline
\end{tabular}

Fonte: Elaboração própria (2016) 
A partir dos riscos identificados, seguiu-se para a análise do risco, nos quais os parâmetros de severidade e frequência de ocorrência foram investigados para priorizar os riscos mais críticos utilizando matrizes de severidade e probabilidade. Após a hierarquização dos riscos, tornou-se possível direcionar qual a medida de mitigação dos riscos poderá ser assumida. A sistemática composta por fases e etapas é esquematizada na Figura 3.

Figura 3 - Sistemática para avaliação dos riscos em etapas e fases

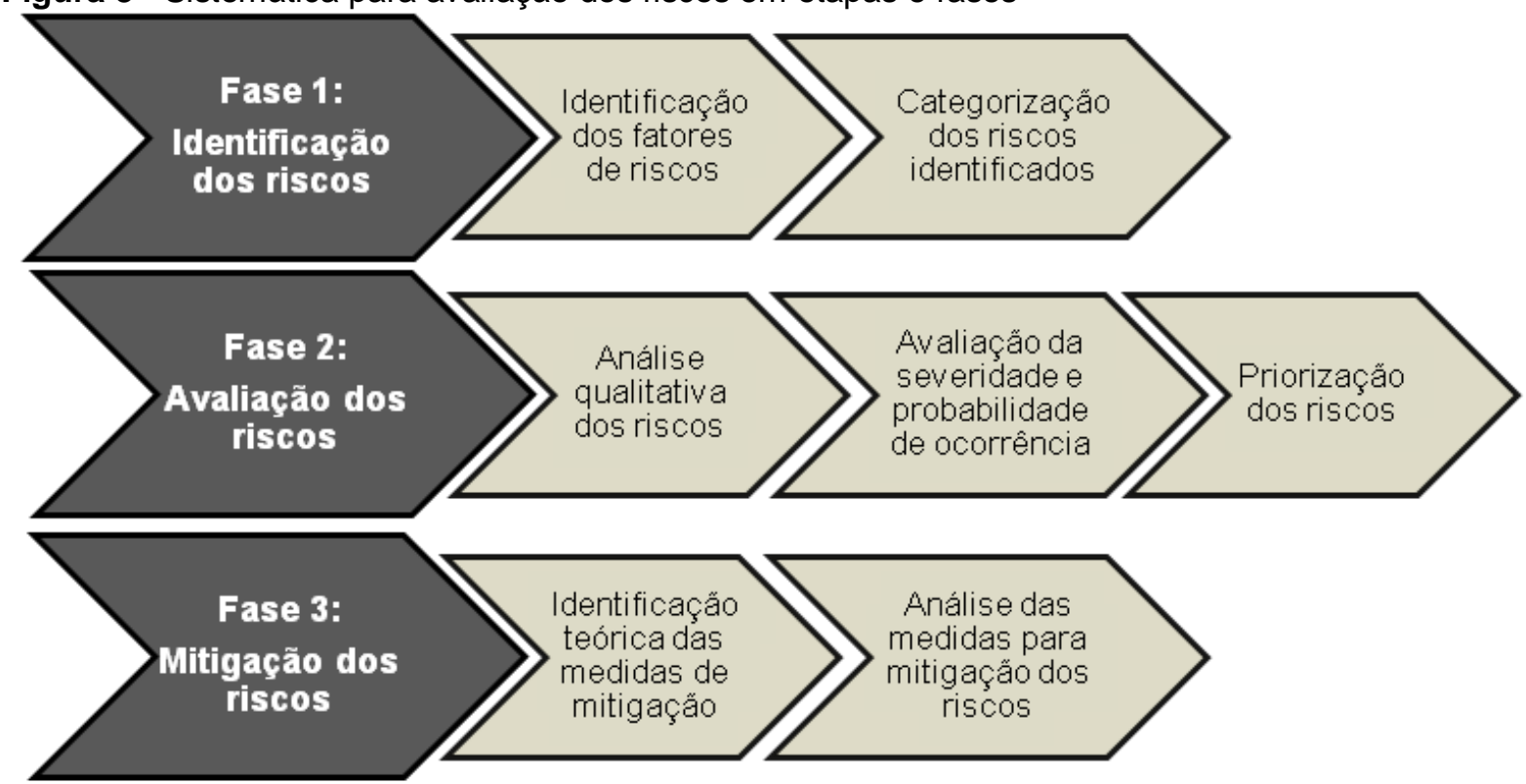

Fonte: Elaboração própria (2016)

Nas duas empresas foram realizadas entrevistas com dois gerentes, que respondiam ao mesmo tempo e assim, ao final da entrevista o pesquisador obtinha um questionário completo respondido. O perfil dos especialistas entrevistados nas empresas é apresentado no Quadro 3.

Pode-se observar que os especialistas que participaram como sujeitos da pesquisa possuem perfil gerencial e com ampla experiência nas empresas investigadas. Isto denota que suas opiniões possuem a coerência esperada sobre o fenômeno pesquisado. 
Quadro 3 - Perfil dos especialistas que participaram das entrevistas

\begin{tabular}{|c|c|c|c|c|}
\hline \multirow{2}{*}{ Empresa } & $\begin{array}{c}\mathbf{N}^{\circ} \text { de } \\
\text { especialistas } \\
\text { entrevistados }\end{array}$ & Função & $\begin{array}{c}\text { Tempo de atuação } \\
\text { na empresa }\end{array}$ & $\begin{array}{c}\text { Grau de } \\
\text { instrução }\end{array}$ \\
\hline \multirow{2}{*}{ A } & 2 & $\begin{array}{c}\text { Gerente de } \\
\text { Qualidade }\end{array}$ & 14 anos & $\begin{array}{c}\text { Engenheiro de } \\
\text { Alimentos }\end{array}$ \\
\cline { 3 - 5 } & \multirow{2}{*}{ B } & $\begin{array}{c}\text { Gerente de } \\
\text { Produção }\end{array}$ & 12 anos & Administrador \\
\cline { 2 - 5 } & 2 & $\begin{array}{c}\text { Diretor Industrial } \\
\text { Gerente de } \\
\text { Qualidade }\end{array}$ & 30 anos & Administrador \\
\hline
\end{tabular}

Fonte: Elaboração própria (2016)

\section{APRESENTAÇÃO E DISCUSSÃO DOS RESULTADOS}

A caracterização completa das empresas é apresentada no Quadro 4.

Quadro 4 - Caracterização das empresa participantes da pesquisa

\begin{tabular}{|c|c|c|c|}
\hline Empresa & $\begin{array}{c}\mathbf{N}^{\circ} \text { de } \\
\text { empregados }\end{array}$ & $\begin{array}{c}\text { Principal } \\
\text { produto }\end{array}$ & Descrição da cadeia de suprimentos \\
\hline A & 1500 & $\begin{array}{c}\text { Processamento } \\
\text { de café moído }\end{array}$ & $\begin{array}{c}\text { Empresa focal totalmente desverticalizada adquire as } \\
\text { principais matérias primas de fornecedores localizados } \\
\text { no sudeste do país }\end{array}$ \\
\hline B & 2000 & $\begin{array}{c}\text { Beneficamento } \\
\text { de carne de } \\
\text { frango }\end{array}$ & $\begin{array}{c}\text { Empresa focal com muitos fornecimentos verticalizados } \\
\text { possuindo serviços de terceiros nos elos a jusante da } \\
\text { cadeia }\end{array}$ \\
\hline
\end{tabular}

Fonte: Elaboração própria (2016)

O resumo dos riscos que foram identificados é sumarizado no Quadro 5 (empresa A) e Quadro 6 (empresa B).

Analisando os Quadros 5 e 6, observa-se que alguns riscos ocorrem nas duas empresas, como por exemplo, risco de segurança, risco de preço, risco de demanda, risco de capacidade, risco operacional e risco de informação. A maioria desses riscos são de natureza interna à empresa, o que denota ainda dificuldade de gestão das empresas pesquisadas.

Apenas os riscos de preço, de mercado e politico para a empresa A e dos riscos de preço e de cliente para a empresa $B$ são de natureza externa à empresa, o que denota dificuldade da empresa em lidar com incertezas externas relacionadas ao comportamento do cliente e dos concorrentes. 
Quadro 5- Matriz com riscos identificados para a empresa A

\begin{tabular}{|c|c|c|}
\hline RISCO & FATORES DOS RISCOS & EFEITO DO RISCO \\
\hline SEGURANÇA & $\begin{array}{l}\text { - Sabotagem de máquinas por } \\
\text { funcionários; } \\
\text { - Acidentes de trabalhos no interior da } \\
\text { empresa }\end{array}$ & $\begin{array}{l}\text { - Maior parada de produção; } \\
\text { - Imagem ruim da empresa; }\end{array}$ \\
\hline POLÍTICO & - Incertezas no ambiente político do país & $\begin{array}{l}\text { - Menor investimento para aumento } \\
\text { da capacidade de produção }\end{array}$ \\
\hline PREÇO & $\begin{array}{l}\text { - Variações no preço das materias primas; } \\
\text { - Escassez da matéria-prima }\end{array}$ & - Maiores custos de produção \\
\hline MERCADO & - Mudanças nas preferências dos clientes & $\begin{array}{l}\text { - Perda de participação no } \\
\text { mercado; }\end{array}$ \\
\hline DEMANDA & $\begin{array}{l}\text { - Aumento inesperado da demanda; } \\
\text { - Erros na previsão de demanda }\end{array}$ & $\begin{array}{l}\text {-Perda de participação no mercado; } \\
\text { - Obsolescência dos produtos; }\end{array}$ \\
\hline CAPACIDADE & $\begin{array}{l}\text { - Limitação na capacidade (disponibilidade) } \\
\text { dos recursos }\end{array}$ & $\begin{array}{l}\text { - Perda de participação no } \\
\text { mercado; }\end{array}$ \\
\hline OPERACIONAL & $\begin{array}{l}\text { - Falhas no planejamento dos serviços de } \\
\text { manutenção; } \\
\text { - Falhas na programação da produção }\end{array}$ & - Maior parada de produção; \\
\hline FORNECIMENTO & $\begin{array}{l}\text { - Problemas no prazo de entregue das } \\
\text { matéria-prima; } \\
\text { - Problemas na qualidade da matéria-prima }\end{array}$ & - Descontinuidade do suprimento; \\
\hline ESTOQUE & $\begin{array}{l}\text { - Falta de itens em estoque; } \\
\text { - Obsolescência insumos estocados }\end{array}$ & - Obsolescência dos produtos; \\
\hline INFORMAÇÃO & $\begin{array}{l}\text { - Indisponibilidade no sistema de } \\
\text { informação }\end{array}$ & $\begin{array}{l}\text { - Dificuldade de gestão; } \\
\text { - Maior parada de produção; }\end{array}$ \\
\hline
\end{tabular}

Fonte: Elaboração própria (2016)

Quadro 6- Matriz com riscos identificados para a empresa B

\begin{tabular}{|c|c|c|}
\hline RISCO & FATORES DOS RISCOS & EFEITO DO RISCO \\
\hline SEGURANÇA & $\begin{array}{l}\text { - Ocorrência de vandalismo; } \\
\text { - Ocorrência de acidentes de trabalho }\end{array}$ & $\begin{array}{l}\text { - Maior parada de produção; } \\
\text { - Imagem ruim da empresa; }\end{array}$ \\
\hline PREÇO & $\begin{array}{l}\text { - Prática de preços variados por } \\
\text { segmentos de clientes }\end{array}$ & $\begin{array}{l}\text { - Perda de participação no } \\
\text { mercado; }\end{array}$ \\
\hline CONTROLE & - Acurácia dos controles & - Perda do controle de qualidade; \\
\hline DEMANDA & $\begin{array}{l}\text { - Aumento inesperado da demanda; } \\
\text { - Erros na previsão de demanda }\end{array}$ & $\begin{array}{l}\text {-Perda de participação no mercado; } \\
\text { - Obsolescência dos produtos; }\end{array}$ \\
\hline CAPACIDADE & $\begin{array}{l}\text { - Inflexibilidade em alterar a capacidade } \\
\text { dos recurso }\end{array}$ & $\begin{array}{l}\text { - Perda de participação no } \\
\text { mercado; }\end{array}$ \\
\hline OPERACIONAL & $\begin{array}{l}\text { - Falhas no planejamento dos serviços de } \\
\text { manutenção; } \\
\text {-Falhas humanas na operabilidade diária. }\end{array}$ & - Maior parada de produção; \\
\hline LOGÍSTICOS & $\begin{array}{l}\text { - Falhas na infra estrutura de transporte } \\
\text { para entregar os produtos finais; } \\
\text { - Perda repentina dos serviços de } \\
\text { terceiros. }\end{array}$ & - Maiores custos de produão; \\
\hline INFORMAÇÃO & $\begin{array}{l}\text { - Grau de usuabilidade do sistema de } \\
\text { informação; } \\
\text { - Acurácia das informações }\end{array}$ & $\begin{array}{l}\text { - Dificuldade de gestão; } \\
\text { - Maior parada de produção; }\end{array}$ \\
\hline CLIENTE & $\begin{array}{l}\text { - Escolha por produtos } \\
\text { similares/substitutos }\end{array}$ & $\begin{array}{l}\text { - Perda de participação no } \\
\text { mercado; } \\
\text { - Obsolência dos produtos }\end{array}$ \\
\hline
\end{tabular}

Fonte: Elaboração própria (2016) 
Observa-se ainda que na empresa $A$, participante de uma cadeia de suprimentos totalmente desverticalizada a montante da cadeia, há a ocorrência de riscos de fornecimento, o que não ocorre na empresa $B$, participante de uma cadeia de suprimentos com parte do fornecimento das matérias primas principais verticalizadas. Como alternativa para vencer as incertezas de fornecimento, a empresa A adota grandes pulmões de estoque de matérias primas, o que justifica o aumento de ocorrência de perda de material por obsolescência.

Da mesma forma, observa-se que na empresa $B$, apesar de não se registrar riscos relativos ao fornecimento, observa-se a ocorrência de riscos relativos à distribuição dos produtos acabados, especificamente os riscos logísticos.

De modo geral, também observa-se que os efeitos mais esperados dizem respeito às paradas não programadas da produção, maiores custos de produção, obsolescência dos estoques e perda de participação no mercado.

Após a identificação dos riscos, procedeu-se a avaliação dos riscos por meio de uma análise qualitativa sobre os riscos. Essa análise foi direcionada por meio de matrizes de probabilidade e impacto, assumindo uma escala para dimensionar as escalas da severidade (entre 1 e 5) e as escalas de frequência de ocorrrência (entre 1 e 3). Isso pode ser observado nas Tabelas 3 e 4.

Tabela 3- Escala para análise da severidade do risco

\begin{tabular}{cc}
\hline ESCALA & CONCEITO \\
\hline 1 & IMPACTO SEVERO \\
2 & IMPACTO ALTO \\
3 & IMPACTO MÉDIO \\
4 & IMPACTO PEQUENO \\
5 & NENHUM IMPACTO \\
\hline Fonte: Elaboração própria (2016)
\end{tabular}

Tabela 4- Escala para análise da frequência de ocorrência do risco

\begin{tabular}{cc}
\hline ESCALA & CONCEITO \\
\hline 1 & FREQUÊNCIA ALTA \\
2 & FREQUÊNCIA MÉDIA \\
3 & FREQUÊNCIA BAIXA \\
\hline
\end{tabular}

Fonte: Elaboração própria (2016) 
Para as categorias de riscos categorizadas, tornou-se necessário investigar os parâmetros de severidade e frequência de ocorrência de riscos e essas análises são apresentadas por meio de matrizes que são ilustradas nas Figuras 4 e 5.

Figura 4 - Matriz de impacto e frequência de ocorrência do riscos na empresa A

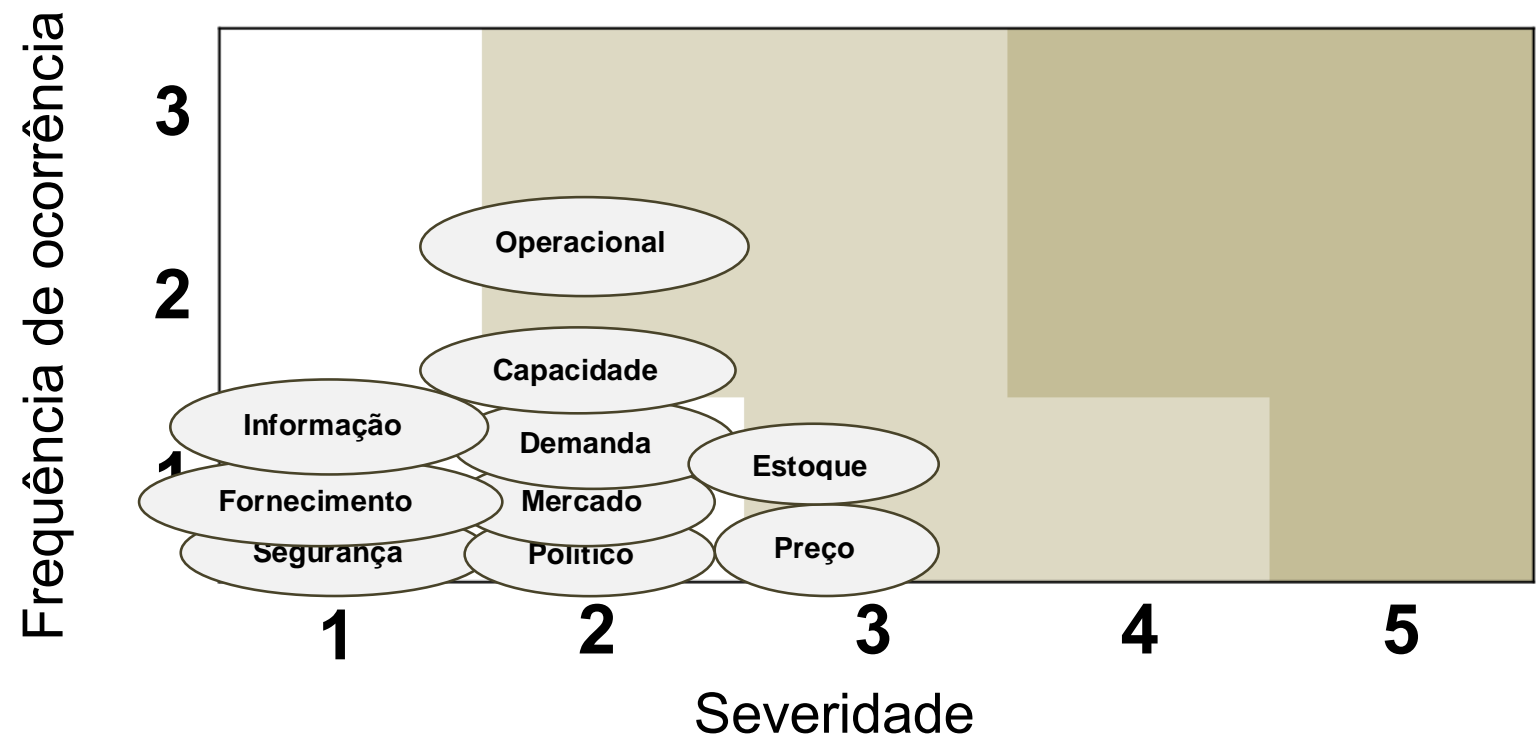

Fonte: Elaboração própria (2016)

Figura 5 - Matriz de impacto e frequência de ocorrência do riscos na empresa B

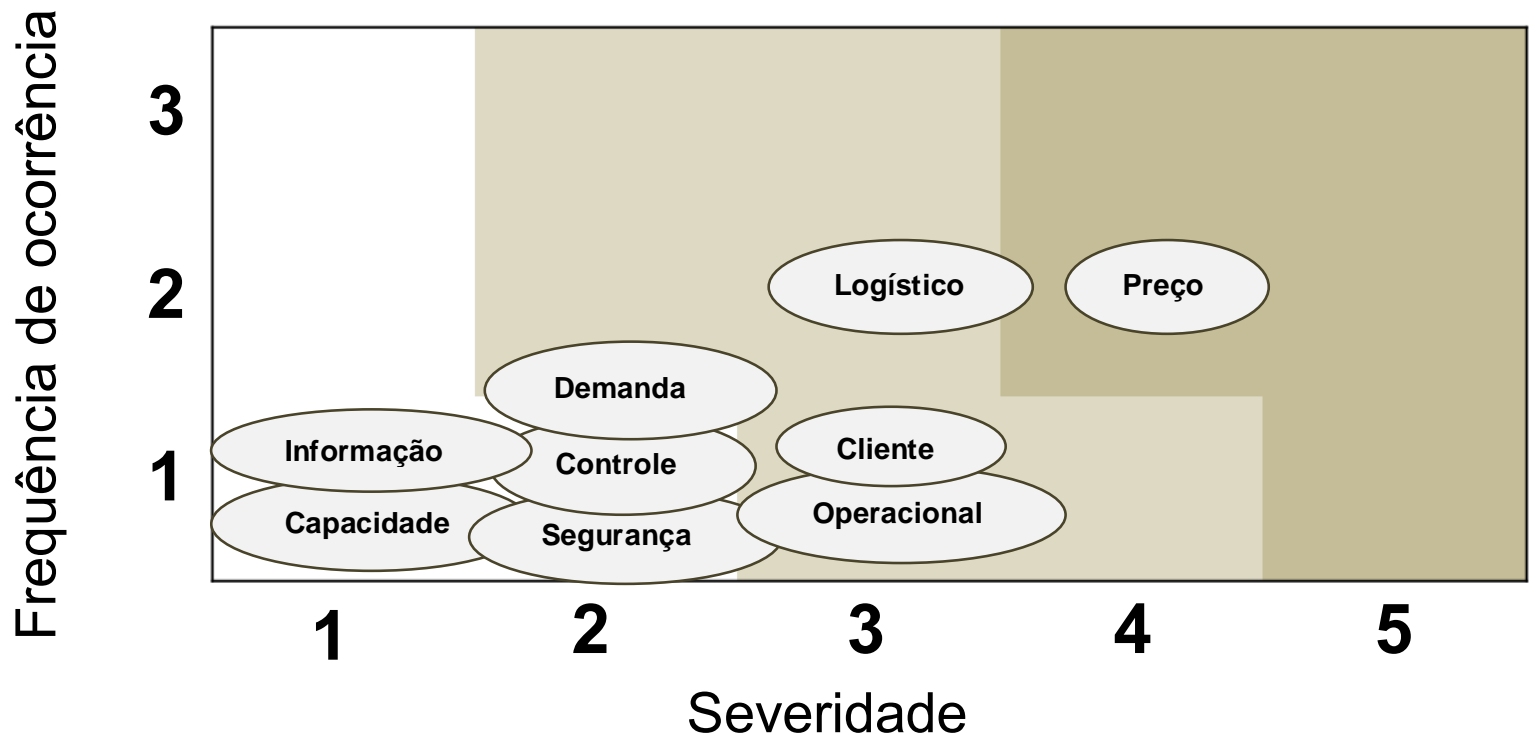

Fonte: Elaboração própria (2016) 
Pela avaliação dos riscos exposta nas matrizes de severidade e frequência de ocorrência é possível priorizar os riscos mais críticos para ambas as empresas. Esta priorização é apresentada nos Quadros 7 e 8.

Quadro 7- Priorização dos riscos por criticidade na empresa $A$

\begin{tabular}{|l|c|c|}
\hline \multicolumn{1}{|c|}{ CRITICIDADE DOS RISCOS } & AÇÃO ESPERADA & TIPOS DE RISCOS \\
\hline RISCOS MAIS CRÍTICOS & Análise para correção & Operacional, estoque e preço \\
\hline RISCOS COM CRITICIDADE MÉDIA & Análise para controle & $\begin{array}{c}\text { Capacidade, demanda, mercado e } \\
\text { político }\end{array}$ \\
\hline RISCOS MENOS CRÍTICOS & Nenhuma ação & $\begin{array}{c}\text { Informação, fornecimento e } \\
\text { segurança }\end{array}$ \\
\hline
\end{tabular}

Fonte: Elaboração própria (2016)

Quadro 8- Priorização dos riscos por criticidade na empresa $B$

\begin{tabular}{|l|c|c|}
\hline \multicolumn{1}{|c|}{ CRITICIDADE DOS RISCOS } & AÇÃO ESPERADA & TIPOS DE RISCOS \\
\hline RISCOS MAIS CRÍTICOS & Ação imediada & Preço e logístico \\
\hline RISCOS MAIS CRÍTICOS & Análise para correção & Cliente e operacional \\
\hline RISCOS COM CRITICIDADE MÉDIA & Análise para controle & Demanda, controle e Segurança \\
\hline RISCOS MENOS CRÍTICOS & Nenhuma ação & Informação e capacidade \\
\hline
\end{tabular}

Fonte: Elaboração própria (2016)

Nos Quadros 7 e 8, observa-se que a depender do grau de criticidade do risco, deve-se adotar uma ação diferenciada por parte das empresas. Deste modo, para a empresa A os riscos que carecem de ação para correção são os riscos operacionais, de estoque e de preço.

Enquanto na empresa $B$ ocorreu um tipo de risco mais crítico que merece uma ação mais imediata que é o risco de preço, pois é o risco com maior grau de severidade. Além disso, na empresa B identificou-se os riscos logísticos, de cliente e operacional como riscos com alta criticidade para os quais é necessário análise para correção.

Por outro lado, alguns riscos foram avaliados como de baixa criticidade para os quais não sugere-se nenhuma ação imediata, sugerindo-se monitoramento a fim de controlá-lo caso haja mudanças nos parâmetros de severidade e frequência de ocorrência. Para a empresa $A$, os riscos com essa baixa criticidade são os riscos de informação, de fornecimento e de segurança. Já na empresa B estes riscos são os riscos de informação e de capacidade. 
Após a hierarquização dos riscos, e com isso a partir da observação das categorias dos riscos que possuem um cárater prioritário para correção, é importante direcionar quais as medidas podem ser assumidas de maneira a mitigar os riscos. Para tal, é importante apontar as estratégias que podem ser adotadas, observando a natureza do risco e com isso o objetivo da medida, se eliminar o risco ou reduzir a severidade relacionada ao risco. O direcionamento das medidas e o objetivo pretendido em relação aos riscos mais críticos das duas cadeias de suprimentos são expostos nos Quadros 9 e 10.

Quadro 9- Medidas de mitigação dos riscos na empresa A

\begin{tabular}{|c|c|c|l|}
\hline RISCOS & NATUREZA DO RISCO & OBJETIVO & AÇÃO DE MITIGAÇÃO \\
\hline Preço & $\begin{array}{c}\text { Externa à empresa e } \\
\text { interna a cadeia }\end{array}$ & $\begin{array}{c}\text { Reduzir a } \\
\text { severidade }\end{array}$ & $\begin{array}{l}\text { - Desenvolver fornecedores; } \\
\text { - Estabelecer contratos de fornecimentos; }\end{array}$ \\
\hline Operacional & Interna à empresa & $\begin{array}{c}\text { Reduzir a } \\
\text { frequência }\end{array}$ & $\begin{array}{l}\text { - Adequar a gestão da manutenção ao } \\
\text { planejamento da produção }\end{array}$ \\
\hline Estoque & Interna à empresa & $\begin{array}{c}\text { Reduzir a } \\
\text { frequência }\end{array}$ & - Melhorar o planejamento da demanda \\
\hline
\end{tabular}

Fonte: Elaboração própria (2016)

Quadro 10- Medidas de mitigação dos riscos na empresa B

\begin{tabular}{|c|c|c|l|}
\hline RISCOS & NATUREZA DO RISCO & OBJETIVO & \multicolumn{1}{|c|}{ AÇÃO DE MITIGAÇÃO } \\
\hline Preço & $\begin{array}{c}\text { Externa à empresa e } \\
\text { interna a cadeia }\end{array}$ & $\begin{array}{c}\text { Reduzir a } \\
\text { severidade }\end{array}$ & $\begin{array}{l}\text { - Estabelecer políticas de produto por } \\
\text { segmento de mercado; }\end{array}$ \\
\hline Logístico & Interna à empresa & $\begin{array}{c}\text { Reduzir a } \\
\text { frequência }\end{array}$ & $\begin{array}{l}\text { - Desenvolver a gestão de terceiros da área } \\
\text { de distribuição; }\end{array}$ \\
\hline Opliente & $\begin{array}{c}\text { Externa à empresa e } \\
\text { interna a cadeia }\end{array}$ & $\begin{array}{c}\text { Reduzir a } \\
\text { severidade }\end{array}$ & $\begin{array}{l}\text { - Desenvolver estratégias para fidelização } \\
\text { dos produtos }\end{array}$ \\
\hline Interna à empresa & $\begin{array}{c}\text { Reduzir a } \\
\text { frequência }\end{array}$ & $\begin{array}{l}\text { - Adequar a gestão da manutenção ao } \\
\text { planejamento da produção } \\
\text { - Desenvolver procedimentos das } \\
\text { operações }\end{array}$ \\
\hline
\end{tabular}

Fonte: Elaboração própria (2016)

Observa-se que a avaliação dos riscos para as empresas participantes das cadeias de suprimentos analisadas, permitiu identificar os riscos que ocorriam nestas empresas, direcionando às empresas para o tratamento mais adequado a ser tomado em função de suas características. Assim, a partir da ação a ser tomada, definir qual área da empresa deve ser mobilizada, determinando processos a serem analisados, responsáveis por estas ações e indicadores a serem monitorados. 
A partir deste direcionamento, é possível estabelecer um plano de monitoramento dos riscos a fim de acompanhar todos os riscos identificados, pois os parâmetros de severidade e frequência podem se alterar e com isso, a criticidade do risco também se altera. Além disso, alguns riscos não identificados inicialmente podem surgir e apenas por meio de monitoramento contínuo é que estes poderão ser visualizadoso que servirá para realimentar o processo de avaliação dos riscos.

\section{CONSIDERAÇÕES FINAIS}

Pelos resultados obtidos no processo de avaliação dos riscos nas duas empresas do setor de alimentos, foi possível observar que mesmo as empresas assumindo características distintas e estando voltadas para produtos diferentes, alguns riscos foram semelhantes em ambas as cadeias de suprimentos.

Dos 24 tipos de riscos investigados o número de riscos externos às cadeias não foram identificados em nenhuma das cadeias. Nesse caso, não se observou a ocorência de riscos ambientais, que ocorrem à revelia das cadeias e das empresas.

No entanto, alguns riscos foram identificados em ambas as empresas. Estes riscos são necessariamente riscos ditos internos, como operacional, de demanda, de capacidade, de informação, por exemplo. Isto denota que as empresas investigadas ainda têm dificuldade de gestão que podem impedir estas empresas a alcançar a máxima eficiência dos recursos produtivos.

Chama atenção a ocorência dos riscos relacionados à atividade de planejamento da produção, notadamente os riscos de demanda e capacidade, denotando a dificuldade enfrentada pelas áreas de planejamento da produção, em definir de maneira mais assertiva a demanda dos produtos e a partir disso planejar adequadamente a capacidade de produção.

Além disso, pode-se observar que a avaliação dos riscos, apesar de qualitativa, mostrou-se eficaz no direcionamento da priorização dos mesmos. No entanto, é importante que quando as empresas adotem um sistema de monitoramento dos riscos, adotem um controle contínuo que permita o levantamento quantitativo da frequência dos riscos. Assim, será possível fazer uma avaliação dos riscos por meio de ferramentas matemáticas mais robustas, o que poderá direcionar de maneira mais eficaz para a mitigação dos riscos. 
Além disso, é importante que os resultados desta pesquisa sejam ampliados investigando às demais empresas que compõem as cadeias de suprimentos investigadas no setor de alimetos. Apenas com esta ampliação será possível generalizar os resutados para toda cadeia de suprimentos.

Sugere-se ainda que trabalhos futuros possam avançar os resultados alcançados modelando um sistema que permita às empresas monitorarem de maneira contínua os riscos, definindo indicadores, medidas e escalas para a probabilidade de ocorrência e severidade do risco. Além disso, sugere-se que trabalhos futuros possam prolongar a identificação e avaliação dos riscos para outros elos da cadeia de suprimentos de forma a verificar quais riscos ocorrem na cadeia e quais estratégias podem ser assumidas pelas empresa de modo a aumentar a resiliência da cadeia como um todo.

Por fim, apesar das limitações pode-se observar que a avaliação dos riscos realizada pode ser utilizada como um primeiro passo para a gestão de riscos nas empresas focais e nas cadeias de suprimentos da qual fazem parte, devendo ser no segundo momento aprimorada de acordo com as necessidades específicas de cada empresa.

\section{REFERÊNCIAS}

ADHITYA, A.; SRINIVASAN, R.; KARIMI, I. Supply chain risk identification using a Hazop based approach. AIChE Journal, v. 55, n. 6, p. 1447 - 1463, 2009.

http://dx.doi.org/10.1002/aic.11764

AHMED, A. et al. A review of techniques for risk management in projects. Benchmarking: An International Journal, v. 14, n 1, p. 22-36, 2007. http://dx.doi.org/10.1108/14635770710730919

AQLAN, F. e LAM, S. Supply chain risk modeling and mitigation. International Journal of Production Research, v. 43, p. 1-17. 2015. http://dx.doi.org/10.1080/00207543.2015.1047975

AQLAN, F. A software application for rapid risk assessment in integrated supply chains. Expert Systems with Applications, v. 43, p. 109-116, 2016. http://dx.doi.org/10.1016/j.eswa.2015.08.028

ASSOCIAÇÃO BRASILEIRA DE NORMAS TÉCNICAS. NBR ISO 31000:2009: Gestão de Riscos: princípios e diretrizes. Rio de Janeiro, 2009.

AUTRY, C. W. BOBBITT, M. Supply chain security orientation: conceptual development and a proposed framework. The Int Journal of Logistics Management, v. 19, n. 1, p. $42-64$. 2008. http://dx.doi.org/10.1108/09574090810872596 
BLACKHURST, J. e SCHEIBE, K. P. e JOHNSON, D. J. Supplier risk assessment and monitoring for the automotive industry. International Journal of Physical Distribution \& Logistics Management, v. 38, n. 2 p. $143-165.2008$.

http://dx.doi.org/10.1108/09600030810861215

BLOME, C. e SCHOENHERR, T. Supply chain risk management in financial crises-A multiple case-study approach. Int. J. Production Economics, v. 134, n 1, p. 43-57. 2011. http://dx.doi.org/10.1016/i.ijpe.2011.01.002

BODE, C. e WAGNER, S. M. Structural drivers of upstream supply chain complexity and the frequency of supply chain disruptions. Journal of Operations Management, v. 36, n. 1,p 215-228. 2015. http://dx.doi.org/10.1016/j.jom.2014.12.004

CERYNO, P; SCAVARDA, L.; KLINGEBIEL, K. Supply chain risk: empirical research in the automotive industry. Journal of Risk Research, v. 18, n. 9, p. 1145 - 1164, 2014. http://dx.doi.org/10.1080/13669877.2014.913662

CHRISTOPHER, M. e LEE, H. Mitigating supply chain risk through improved confidence. Int Journal of Physical Distribution e Logistics Management, v. 34, n. 5, p. 388-396. 2004. http://dx.doi.org/10.1108/09600030410545436

COHEN, M. A e KUNREUTHER, H. Operations Risk Management: Overview of Paul Kleindorfer's Contribution Production and Operations Management. Production and Operations Management Society, v. 16, n. 5, p. 525-541, 2007.

http://dx.doi.org/10.1111/j.1937-5956.2007.tb00278.x

FAZLI, S, MAVI, R. K. e VOSOOGHIDIZAJI, M. Crude oil supply chain risk management with DEMATEL-ANP. Operational Research Int J, p. 1-28. 2015

http://dx.doi.org/10.1007/s12351-015-0182-0

GAONKAR, R. e VISWANADHAM, N. A conceptual and analytical framework for the management of risks in supply chains", Proceedings of the 2004 IEEE International Conference on Robotics and Automation, April 26-May 1, v. 3, p. 2699-704. 2004. http://dx.doi.org/10.1109/ROBOT.2004.1307468

GIANNAKIS, M. e LOUIS, M. A multi-agent based framework for supply chain risk management. Journal of Purchasing \& Supply Management, v. 17, p. 23-31. 2011. http://dx.doi.org/10.1016/j.pursup.2010.05.001

HALLIKAS, J.; et al Risk management processes in supplier networks. International Journal of Production Economics, v. 90, p. 47 - 58, 2004.

http://dx.doi.org/10.1016/j.ijpe.2004.02.007

HARLAND, C., BRENCHLEYB, R. WALKER, H. Risk in supply networks. Journal of Purchasing \& Supply Management, v. 9, p-51-62. 2003. http://dx.doi.org/10.1016/S14784092(03)00004-9

HAUSER, L.M. Risk adjusted supply chain management. Supply Chain Management Review, v. 7, n 6, p. 64-71. 2003.

HO, W. et al. Supply chain risk management: a literature review. International Journal of Production Research, v.53, n. 16, p. 5031-5069. 2015.

http://dx.doi.org/10.1080/00207543.2015.1030467 
JÜTTNER, U. Supply chain risk management: understanding the business requirements from a practitioner perspective. The Int Journal of Logistics Management, v.16, n. 1, p. 120-141, 2005. http://dx.doi.org/10.1108/09574090510617385

JÜTTNER, U.; PECK, H.; CHRISTOPHER, M. Supply chain risk management: outlining an agenda for future research. Int Journal of Logistics: Research \& Applications, v. 6, n. 4, p. 197-210, 2003. http://dx.doi.org/10.1080/13675560310001627016

KAYIS, B. e KARNINGSIH, P. D. A knowledge-based system tool for assisting manufacturing organizations in identifying supply chain risks. Journal of Manufacturing Technology Management, v. 23, n. 7, p. 834-852, 2012. http://dx.doi.org/10.1108/17410381211267682

KERN, D. et al. Supply risk management: model development and empirical analysis.

International Journal of Physical Distribution \& Logistics Management, v. 42, n. 1, p. 60-82, 2012. http://dx.doi.org/10.1108/09600031211202472

KHAN, O. e BURNES, B. Risk and supply chain management: creating a research agenda. The International Journal of Logistics Management, v. 18, n 2, 2007.

http://dx.doi.org/10.1108/09574090710816931

KUMAR, S. HIMES, J.K. e KRITZER, P. C. Risk assessment and operational approaches to managing risk in global supply chains. Journal of Manufacturing Technology

Management, v. 25, n. 6, p. 873 - 890, 2014. http://dx.doi.org/10.1108/JMTM-04-2012-0044

LOCKAMY, III A.e MCCORMACK, K.. Analyzing risks in supply networks to facilitate outsourcing decisions. International Journal of Production Research, v. 48, n. 2, p. 593611. 2010 http://dx.doi.org/10.1080/00207540903175152

MANUJ, I.; MENTZER, J. T. Global supply chain risk management strategies. International Journal of Physical Distribution e Logistics Management, v. 38, n. 3, p. 192-223, 2008.

http://dx.doi.org/10.1108/09600030810866986

MATOOK,S. LASCH, R. e TAMASCHKE,R. Supplier development with benchmarking as part of a comprehensive supplier risk management framework. International Journal of Operations \& Production Management, v. 29, n. 3, p. 241-267, 2009.

http://dx.doi.org/10.1108/01443570910938989

NORRMAN, A.; JANSSON, U. Ericsson's proactive supply chain risk management approach after a serious sub-supplier accident. International Journal of Physical Distribution \& Logistics Management, v. 34, n. 5, p. 434 - 456, 2004.

OLSON, D. L. e WU, D. Risk management models for supply chain :a scenario analysis of outsourcing to China. Supply Chain Management: An Int Journal, v.16, n. 6, 401-408, 2011. http://dx.doi.org/10.1108/13598541111171110

PFOHL, H. C.; GALLUS, P. e THOMAS, D. Interpretive structural modeling of supply chain risks. Intl Journal of Physical Distribution \& Logistics Management, v.41, n. 9, p. 839-859, 2011. http://dx.doi.org/10.1108/09600031111175816

PUNNIYAMOORTHY, M.; THAMARAISELVAN, N.; MANIKANDAN,L. Assessment of supply chain risk: scale development and validation. Benchmarking: An Int Journal, v.20, n.1, p. 79 - 105, 2013. http://dx.doi.org/10.1108/14635771311299506 
PURDY, G. ISO 31000:2009-Setting a New Standard for Risk Management. Risk Analysis. v. 30, n. 6, 2010. http://dx.doi.org/10.1111/j.1539-6924.2010.01442.x

RAO, S.; GOLDSBY, T. Supply chain risks: a review and typology. The International Journal of Logistics Management, v. 20, n. 1, p. 97-123, 2009.

http://dx.doi.org/10.1108/09574090910954864

RITCHIE, B.; BRINDLEY, C. An Emergent Framework for Supply Chain Risk Management and Performance Measurement. The Journal of the Operational Research Society, v. 58, n. 11, p. 1398-1411, 2007b. http://dx.doi.org/10.1057/palgrave.jors.2602412

RITCHIE, B.; BRINDLEY, C. Supply chain risk management and performance: A guiding framework for future development. Int Journal of Operations \& Production Management, v. 27, n. 3, p. 303-322, 2007a. http://dx.doi.org/10.1108/01443570710725563

SODHI, M.; SON, B.; TANG, C. Researchers' Perspectives on Supply Chain Risk Management. Production and Operations Management, v. 21, n. 1, p. 1-13, 2012. http://dx.doi.org/10.1111/j.1937-5956.2011.01251.x

SUN, J., MATSUI, M. e YIN, Y. Supplier risk management: An economic model of P-hart considered due-date and quality risks. International Journal Production Economics, v. 139, p. 58-64, 2012. http://dx.doi.org/10.1016/j.ijpe.2012.03.004

TANG, C. S. Perspective in supply chain risk management. International Journal of production economics, v.103, p. 451-488, 2006.

TANG, O. e MUSA, S. N. Identifying risk issues and research advancements in supply chain risk management. International Journal Production Economics, v.133, p 25-34, 2011. http://dx.doi.org/10.1016/j.ijpe.2010.06.013

TOMAS, R. N. e ALCANTARA, R. L. C. Modelos para gestão de riscos em cadeias de suprimentos: revisão, análise e diretrizes para futuras pesquisas. Gestão e Produção, São Carlos, v. 20, n. 3, p. 695-712, 2013. http://dx.doi.org/10.1590/S0104-530X2013000300014

TRKMAN, P. e McCORMACK, K. Supply chain risk in turbulent environments: a conceptual model for managing supply chain network risk. International Journal Production

Economics, v. 119, p. 247-258, 2009. http://dx.doi.org/10.1016/j.ijpe.2009.03.002

TUMMALA, R. e SCHOENHERR, T. Assessing and managing risks using the Supply Chain Risk Management Process (SCRMP). Supply Chain Management: An International Journal, v.16, n. 6, p. 474-483, 2011. http://dx.doi.org/10.1108/13598541111171165

WANG, D.; YANG, Z.. Risk Management of global supply chain. International Conference on automation and logistics, Jinan, China, august 18-21, 2007.

WIELAND, A. Selecting the right supply chain based on risks. Journal of Manufacturing Technology Management, v. 24, n. 5, p. $652-668,2013$.

http://dx.doi.org/10.1108/17410381311327954

WIENGARTEN, F. et al. Risk, risk management practices, and the success of supply chain integration. International Journal Production Economics, v. 171, p. 361 - 370, 2016. http://dx.doi.org/10.1016/j.ijpe.2015.03.020 
WU. D. e OLSON, D. L. Supply chain risk, simulation, and vendor selection. International Journal Production Economics, v.114, n. 1, p. 646 - 655, 2008.

http://dx.doi.org/10.1016/j.ijpe.2008.02.013

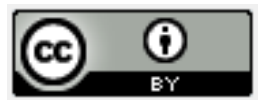

Artigo recebido em 12/08/2016 e aceito para publicação em 01/11/2016

DOI: http://dx.doi.org/10.14488/1676-1901.v17i1.2559 